\title{
Formation of transient high- $\beta$ plasmas in a magnetized, weakly collisional regime
}

\author{
T. Byvank ${ }^{\circledR 1} \uparrow$, D. A. Endrizzi ${ }^{\circledR 2}$, C. B. Forest ${ }^{2}$, S. J. Langendorf ${ }^{1}$, \\ K. J. McCollam ${ }^{2}$ and S. C. Hsu ${ }^{1}$ \\ ${ }^{1}$ Physics Division, Los Alamos National Laboratory, Los Alamos, NM 87545, USA \\ ${ }^{2}$ Wisconsin Plasma Physics Laboratory, Department of Physics, University of Wisconsin, \\ Madison, WI 53706, USA
}

(Received 30 June 2020; revised 13 November 2020; accepted 16 November 2020)

We present experimental data providing evidence for the formation of transient $(\sim 20 \mu \mathrm{s})$ plasmas that are simultaneously weakly magnetized (i.e. Hall magnetization parameter $\omega \tau>1$ ) and dominated by thermal pressure (i.e. ratio of thermal-to-magnetic pressure $\beta>1$ ). Particle collisional mean free paths are an appreciable fraction of the overall system size. These plasmas are formed via the head-on merging of two plasmas launched by magnetized coaxial guns. The ratio $\lambda_{\text {gun }}=\mu_{0} I_{\text {gun }} / \psi_{\text {gun }}$ of gun current $I_{\text {gun }}$ to applied magnetic flux $\psi_{\text {gun }}$ is an experimental knob for exploring the parameter space of $\beta$ and $\omega \tau$. These experiments were conducted on the Big Red Ball at the Wisconsin Plasma Physics Laboratory. The transient formation of such plasmas can potentially open up new regimes for the laboratory study of weakly collisional, magnetized, high- $\beta$ plasma physics; processes relevant to astrophysical objects and phenomena; and novel magnetized plasma targets for magneto-inertial fusion.

Key words: plasma properties, plasma dynamics

\section{Introduction}

Weakly collisional plasmas (particle mean free paths $\lesssim$ system size) with ratio of thermal-to-magnetic pressure $\beta>1$ and, simultaneously, Hall magnetization parameter $\omega \tau>1$ (i.e., gyro-frequency greater than collision frequency), represent a frontier regime of laboratory plasma physics research. By contrast, magnetically confined plasmas typically have $\beta \ll 1$ and $\omega \tau \gg 1$, whereas inertially confined and high-energy-density plasmas typically have $\beta \gg 1$ and $\omega \tau \ll 1$. If plasmas with both $\beta, \omega \tau>1$ can be formed successfully, an interesting next step will be to attempt to generate small-scale, tangled magnetic field with connection length much longer than the characteristic scale size of the plasma. This paper focuses on the first step of forming and characterizing a transient plasma with both $\beta>1$ and $\omega \tau>1$ in a laboratory setting.

One motivation for this work is to help establish a new laboratory platform to study the fundamental physics of weakly collisional, magnetized, high- $\beta$ plasmas (Kunz et al.

$\dagger$ Email address for correspondence: tbyvank@lanl.gov 
2020) as a foundational aspect of the scientific discipline of plasma physics. These types of plasmas, while ubiquitous in the universe, are poorly understood in terms of their basic stability and transport properties (Chandran \& Cowley 1998; Schekochihin et al. 2008), on both macroscales and microscales. A better predictive understanding of the behaviour of these types of plasmas could potentially shed light on the dynamics and evolution of magnetohydrodynamic (MHD) turbulence (Schekochihin \& Cowley 2007; Schekochihin et al. 2009) and magnetostatic turbulence (Ryutov \& Remington 2002; Ryutov et al. 2004), which are fundamentally different compared with the drift-wave turbulence of low- $\beta$, magnetically confined plasmas; on astrophysical systems such as accretion flows around black holes (Balbus \& Hawley 1998), the intracluster medium (known as ICM) within galaxy clusters (Schekochihin et al. 2005; Peterson \& Fabian 2006), the interstellar medium where large-scale structures form (Scalo \& Elmegreen 2004; McKee \& Ostriker 2007), stellar/solar winds (Bruno \& Carbone 2013) and on outstanding cosmological questions such as the origin (magnetogenesis) and amplification (dynamo) of magnetic fields (Kulsrud \& Zweibel 2008). In these systems, although the magnetic pressure is significantly less than the plasma thermal pressure, the magnetic fields can still influence plasma dynamics.

A second motivation for this work is to discover how to form a novel, magnetized, $\beta>1$ target plasma for magneto-inertial fusion (MIF), also known as magnetized target fusion (known as MTF) (Kirkpatrick, Lindemuth \& Ward 1995; Lindemuth \& Siemon 2009). Magneto-inertial fusion is a class of pulsed fusion approaches, where a liner compresses a magnetized target plasma, in which the magnetic field reduces thermal transport and enhances fusion-charged-product (e.g. $\alpha$ particles) energy deposition within the plasma fuel. Many MIF efforts over several decades have focused on the use of $\beta \leq 1$ plasmas, e.g. spheromaks (Bellan 2000) and field-reversed configurations (FRC) (Steinhauer 2011), as the target plasma for subsequent liner compression. However, these $\beta \leq 1$ plasmas suffer from MHD instabilities that have precluded the attainment of robust fusion conditions. This has motivated the consideration of magnetized plasmas that can avoid MHD instabilities while still benefitting from magnetic thermal insulation (Ryutov 2009; Hsu \& Langendorf 2019), which could potentially be enabled by the $\beta, \omega \tau>1$ regime. The lifetime of a $\beta>1$ plasma target will be limited largely by hydrodynamic expansion rather than MHD instability growth, and thus fast liner compression is required, which has been demonstrated successfully in magnetized liner inertial fusion (known as MagLIF) (Slutz et al. 2010; Gomez et al. 2014) and is the aim of plasma-jet-driven MIF (known as PJMIF) (Thio et al. 1999; Hsu et al. 2012a; Thio et al. 2019). The present work is a first step toward determining the viability of forming potential magnetized target plasmas with $\beta, \omega \tau>1$ that may be suitable for subsequent, fast liner compression.

In this work, performing experiments on the Big Red Ball (BRB) (Forest et al. 2015) at the Wisconsin Plasma Physics Laboratory (WiPPL), we launch and merge two $\beta \sim$ 1 plasmas to transiently create $\beta, \omega \tau>1$ conditions for $\sim 20 \mu \mathrm{s}$, and experimentally measure the plasma parameters in both the individual and merged plasmas. Compared with individual plasmas, the head-on collisions (i) increase the duration for which the desired plasma state exists at a particular location and (ii) increase the magnitudes of the density and radial and toroidal magnetic field components, which widens the parameter space compared with that achievable with individual plasmas. The transient nature is unavoidable for $\beta>1$ plasmas unless they are wall confined, which would bring in complications including plasma-wall interactions and impurities. Our plasma-formation approach is analogous to many prior studies that generated $\beta<1$ spheromaks using magnetized coaxial guns (Bellan 2000) and the merging/collision of two $\beta<1$ compact toroids, e.g. spheromaks (Bellan 2000) or field reversed configurations (FRC) (Steinhauer 
2011), for magnetic-reconnection studies (Yamada et al. 1990; Ono et al. 1999; Cothran et al. 2003) and fusion concept exploration (Guo et al. 2011; Slough, Votroubek \& Pihl 2011). To access various portions of the $\beta, \omega \tau$ parameter space, we tune the experimentally adjustable parameter $\lambda_{\text {gun }}=\mu_{0} I_{\text {gun }} / \psi_{\text {gun }}$, where $I_{\text {gun }}$ is the peak gun electrical current and $\psi_{\text {gun }}$ is the applied vacuum poloidal magnetic flux linking the gun electrodes (Yee \& Bellan 2000; Hsu \& Bellan 2005). Engineering improvements to our plasma injectors are presently underway to improve reliability and reproducibility of plasma formation.

The paper is organized as follows. Section 2 provides background information on the scaling of $\beta$ and $\omega \tau$ and the plasma-formation process. Section 3 describes the experimental set-up and diagnostics. Section 4 presents experimental results for individual and merged plasmas. Section 5 discusses future work. Section 6 provides conclusions.

\section{Background}

In this section, we provide background information that further motivates this work and helps with understanding the experimental set-up, methods and the results presented later in the paper.

\subsection{Plasma parameters}

In this subsection, we describe the plasma parameters needed to achieve $\beta, \omega \tau>1$ simultaneously. The definition of $\beta$ is

$$
\beta=\frac{P_{\mathrm{th}}}{P_{\mathrm{mag}}}=\frac{2 \mu_{0} \sum_{j} n_{j} k_{B} T_{j}}{B^{2}} \approx\left(4.0 \times 10^{-11}\right) \frac{\sum_{j} n_{j}\left[\mathrm{~cm}^{-3}\right] T_{j}[\mathrm{eV}]}{(B[\mathrm{G}])^{2}} \propto \frac{n_{i}\left(Z T_{e}+T_{i}\right)}{B^{2}},
$$

where $P_{\text {th }}$ is the plasma thermal pressure, $P_{\text {mag }}$ the magnetic pressure, $\mu_{0}$ the vacuum permeability, $n_{j}$ the ion or electron density $(j=i, e), k_{B}$ the Boltzmann constant, $T_{j}$ the ion or electron temperature, $B$ the magnetic field strength and $Z$ the mean ion charge state. A thermal-pressure-dominated plasma has $\beta>1$. The definitions of the ion and electron Hall magnetization parameters, $\omega_{i} \tau_{i}$ and $\omega_{e} \tau_{e}$, respectively, are

$$
\omega_{i} \tau_{i}=\frac{\omega_{i}}{\nu_{i}} \approx\left(2.0 \times 10^{11}\right) \frac{B[\mathrm{G}]\left(T_{i}[\mathrm{eV}]\right)^{3 / 2}}{Z^{3} \mu^{1 / 2} n_{i}\left[\mathrm{~cm}^{-3}\right] \ln \Lambda} \propto \frac{B T_{i}^{3 / 2}}{Z^{3} \mu^{1 / 2} n_{i}}
$$

and

$$
\omega_{e} \tau_{e}=\frac{\omega_{e}}{v_{e}} \approx\left(6.0 \times 10^{12}\right) \frac{B[\mathrm{G}]\left(T_{e}[\mathrm{eV}]\right)^{3 / 2}}{n_{e}\left[\mathrm{~cm}^{-3}\right] \ln \Lambda} \propto \frac{B T_{e}^{3 / 2}}{n_{e}},
$$

where $\omega_{i, e}$ are the ion and electron gyrofrequencies, $\tau_{i, e}$ the ion and electron collision times, $v_{i, e}$ the ion and electron collision rates, $\mu$ the atomic mass number and $\ln \Lambda \approx 10$ the Coulomb logarithm. The condition $\omega_{i} \tau_{i}>1$ is usually more stringent than $\omega_{e} \tau_{e}>1$.

Figure 1 illustrates contours of $\beta, \omega_{i} \tau_{i}=0.1,1$ and 10 as a function of $n, T$ and $B$, assuming that $n=n_{i}=n_{e}$ (and therefore $Z=1$ ), $T=T_{i}=T_{e}$ and $\mu=1$. The shaded regions in the plots denote the parameter spaces where $\beta, \omega_{i} \tau_{i}>1$. Because $n$ is in the numerator of $\beta$ and denominator of $\omega \tau$, and vice versa for $B$, in general there are only limited ranges and combinations of $n, T$ and $B$ for which $\beta$ and $\omega \tau$ can be simultaneously greater than unity. In general, higher $T$ helps because $T$ is in the numerator for both $\beta$ and $\omega \tau$. Additionally in figure 1 , we plot the ratio of the ion cyclotron radius $\rho_{c i} \propto T_{i}^{1 / 2} / B$ to the characteristic plasma radius $L_{0}=R=30 \mathrm{~cm}$, here. The dashed arrow shows the region in parameter space for which the characteristic plasma radius is larger than the 

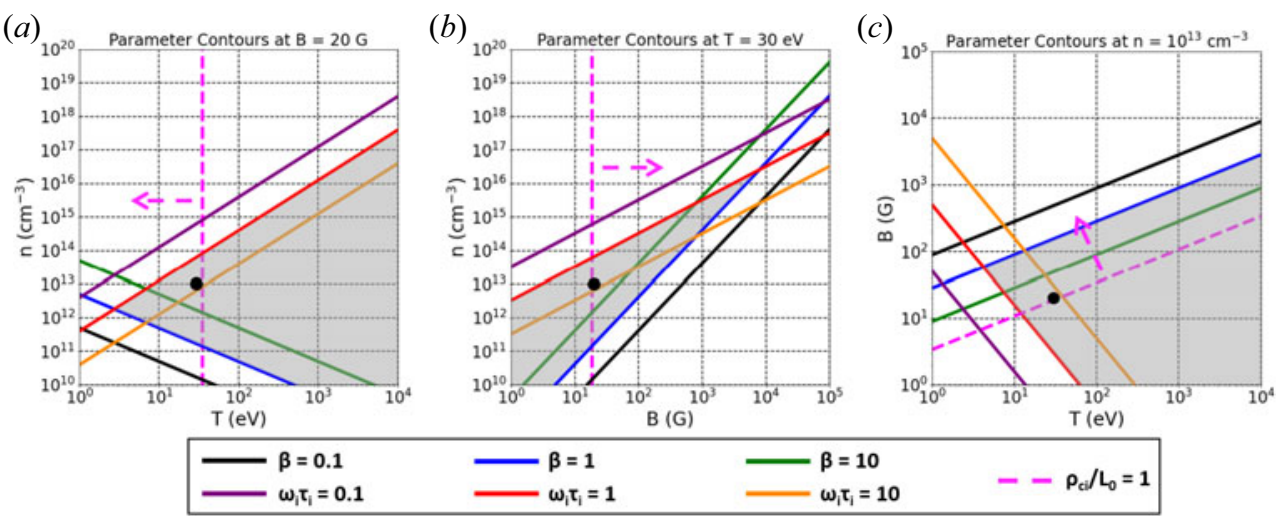

Figure 1. Contours of $\beta, \omega_{i} \tau_{i}$ and $\rho_{c i} / L_{0}$ in the (a) $n-T$ plane at constant $B=20 \mathrm{G},(b) n-B$ plane at constant $T=T_{i}=T_{e}=30 \mathrm{eV}$ and $(c) B-T$ plane at constant $n=n_{i}=n_{e}=10^{13} \mathrm{~cm}^{-3}$. The shaded regions correspond to the desired regime of $\beta, \omega \tau>1$. The dashed arrows show the regime for which $\rho_{c i} / L_{0}<1$, with $L_{0}=30 \mathrm{~cm}$. The dots correspond to the approximate values obtained in the present research.

ion cyclotron radius, $\rho_{c i} / L_{0}<1$. The dots in the plots denote approximately the values obtained experimentally in this research.

\subsection{Plasma formation}

In this subsection, we describe the plasma-formation method and insights derived from previous work that we exploit to achieve the objectives of the present research. It has long been known that compact-toroid plasmas, i.e. spheromaks and FRCs, are formed in the $\beta \leq 1, \omega \tau>1$ regime, where the thermal pressure is confined by an equal or larger magnetic pressure.

Spheromak and spheromak-like plasmas can be created using magnetized plasma guns with coaxial, cylindrical electrodes in the presence of an applied 'bias' poloidal magnetic flux $\psi_{\text {gun }}=\int B_{\text {pol }} \cdot \mathrm{d} A$ linking the two electrodes (see figure 2). Electrical current $I_{\text {gun }}$ in the electrodes ( $z$ direction) and in the plasma ( $r$ direction) that forms between the electrodes generates a toroidal magnetic field ( $B_{\text {tor }}$, in the $\phi$ direction), and the associated magnetic pressure accelerates the plasma out of the electrodes ( $z$ direction). The moving plasma advects the poloidal bias magnetic field $\left(B_{\mathrm{pol}}\right.$, in the $r-z$ plane). Depending on the values of $I_{\text {gun }}$ and $\psi_{\text {gun }}$, the magnetic field lines associated with the plasma can reconnect and detach from the electrodes as the plasma propagates out of the gun. The resulting plasma has both (i) $B_{\text {pol }}$ associated with toroidal currents and (ii) $B_{\text {tor }}$ associated with poloidal currents. The relative values of magnetic field, density and temperature in the plasma, and their evolution, determine the values of $\beta$ and $\omega \tau$. For $\beta>1$, the magnetic field is not large enough to create an equilibrium with the plasma thermal pressure, so $\beta>1$ plasmas will exist only transiently.

The $\lambda_{\text {gun }}$ parameter,

$$
\lambda_{\text {gun }}=\frac{\mu_{0} I_{\text {gun }}}{\psi_{\text {gun }}} \propto \frac{B_{\text {tor }}}{\int B_{\text {pol }} \cdot \mathrm{d} A},
$$

determines whether a spheromak forms (Bellan 2000; Yee \& Bellan 2000; Hsu \& Bellan 2005). A spheromak $(\beta<1, \omega \tau>1)$ will be formed under the condition (Bellan 2000)

$$
\lambda_{\text {gun }}>\lambda_{\text {crit }} \approx 3.83 / r_{\text {gun }}
$$




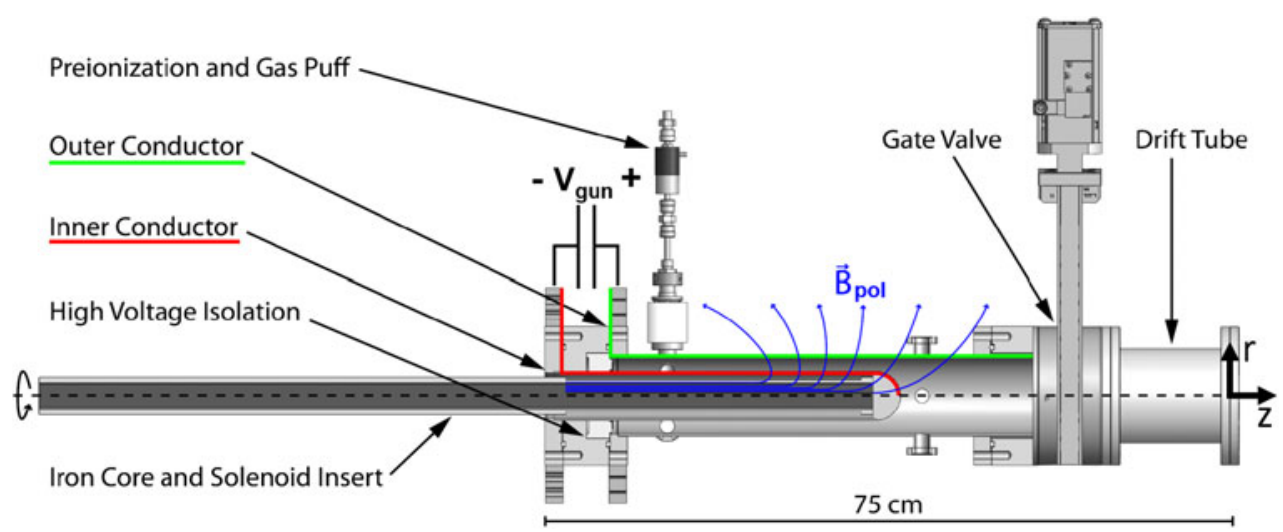

FIGURE 2. Diagram of a plasma gun/injector used in this research, showing the coordinate system, inner (cathode, red) and outer (anode, green) electrodes, and solenoid and iron core that generate the bias poloidal magnetic flux (blue). Gas is injected between the electrodes and preionized using a separate 'washer gun' system (Fiksel et al. 1996). Finally, a discharge with gun voltage $V_{\text {gun }}$ between the electrodes accelerates the plasma out of the injector in the $z$ direction.

where 3.83 is the first root of the Bessel function of the first kind $J_{1}(x)$, and $r_{\text {gun }}$ is the characteristic size (e.g. inner radius of the outer gun electrode). On the other hand, a plasma jet with rapidly decaying magnetic flux is formed when $\psi_{\text {gun }}=0$ and $\lambda_{\text {gun }}=\infty$ (Hsu et al. 2012 b; Merritt et al. 2014), resulting in a plasma with $\beta \gg 1$ and $\omega \tau \ll 1$ after a few resistive decay times of the magnetic flux (of the order of several $\mu$ ). Given these two bracketing conditions, it is intuitive to expect that a plasma with both $\beta, \omega \tau>1$ may occur for some $\lambda_{\text {gun }}$ satisfying $\lambda_{\text {crit }}<\lambda_{\text {gun }}<\infty$.

In the present work, we launch and collide (head-on) two plasmas formed by coaxial guns. We orient the applied bias magnetic fields such that the plasmas have opposite magnetic helicities $H$, where $H=\int \boldsymbol{A} \cdot \boldsymbol{B} \mathrm{d} V$ and $\boldsymbol{A}$ is the magnetic vector potential with $B=\nabla \times A$. In the $\lambda_{\text {gun }} \gtrsim \lambda_{\text {crit }}$ regime, the helicity of merging compact-toroid plasmas determines the magnetic topology of the resultant plasma, i.e. the merging of two cohelicity or counter-helicity spheromaks creates a $\beta \ll 1$ spheromak or a $\beta \lesssim 1$ FRC, respectively (Yamada et al. 1990; Ono et al. 1999). However, this precludes the achievement of the $\beta, \omega \tau>1$ regime that we seek. The goal of this research is to explore and characterize the head-on merging of two coaxial-gun-formed plasmas in the range $\lambda_{\text {crit }}<\lambda_{\text {gun }}<\infty$ in order to form a transient plasma with $\beta, \omega \tau>1$.

\subsection{Plasma propagation and merging}

As the plasmas propagate into the vacuum chamber from the magnetized coaxial guns, they expand into an applied background magnetic field that is oriented along the propagation direction ( $z$ axis). The applied background field mitigates the amount of expansion. To estimate the range of plasma parameters during plasma propagation, we use a combination of measurements at the chamber centre and near the gun, along with basic scaling relationships. This expected range of plasma parameters dictates the range of $\beta, \omega \tau$, Mach number $M$, Alfvén Mach number $M_{A}$ and mean free paths, as well as the nature of the plasma-merging dynamics. Further details are provided in $\S 4.1$. 


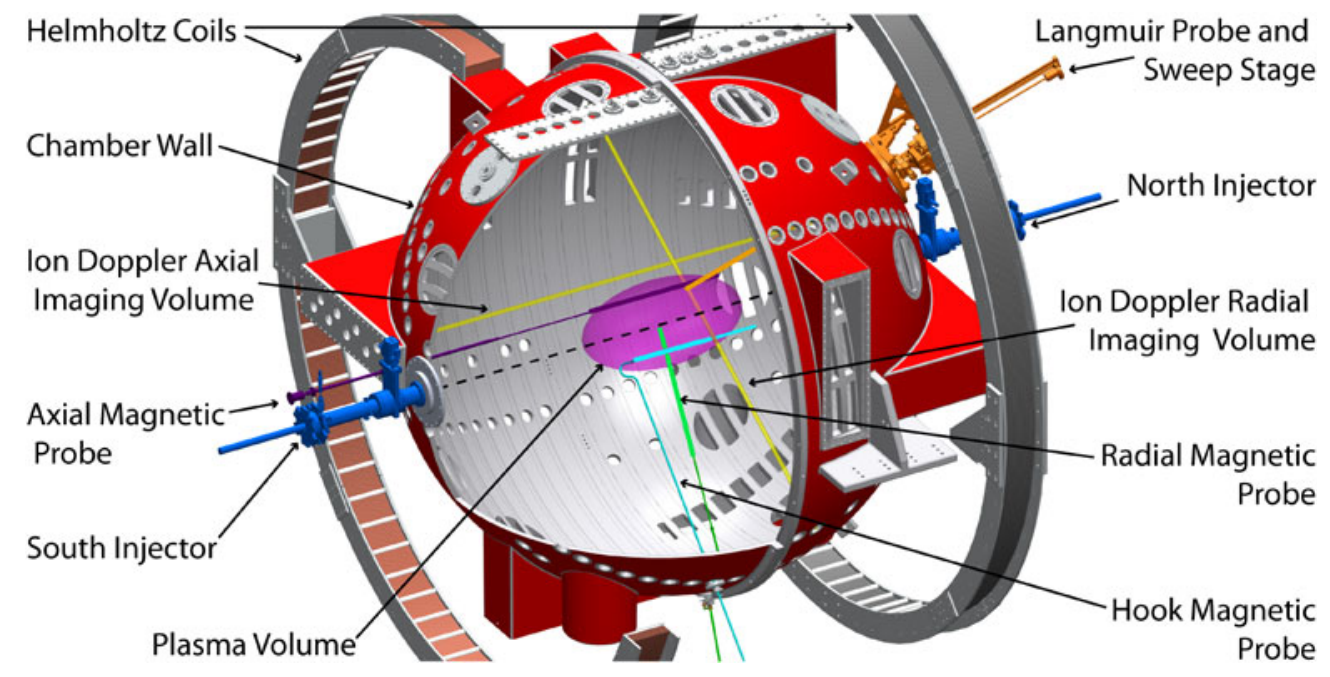

FIGURE 3. Diagram of the experimental set-up, showing relative locations of the plasma injectors, diagnostics, approximate plasma volume in the $3 \mathrm{~m}$ diameter BRB chamber, and external Helmholtz coils.

\section{Experimental setup}

In this work, we launch two $\beta>1$ plasmas head-on within a vacuum chamber and measure the resulting plasma densities, temperatures and magnetic fields. The experiments are conducted on the BRB, which is part of the WiPPL, a frontier-plasma-science user facility.

\subsection{Experimental chamber: the $B R B$}

Figure 3 shows a diagram of the BRB experimental chamber at the WiPPL (Cooper et al. 2014; Forest et al. 2015). The multicusp magnetic confinement of the BRB contributes $<1 \mathrm{G}$ to the plasma-merging region. Two plasma injectors are positioned on opposite poles (labelled south and north) of the $3 \mathrm{~m}$ diameter vacuum chamber at a base pressure of $\sim 4 \mu$ Torr. A $3 \mathrm{~m}$ diameter Helmholtz coil set outside of the chamber provides a 50 $\mathrm{G}$ (direct current) magnetic field pointing from south to north. This background magnetic field serves to prevent the $\beta>1$ plasmas from expanding too much and reducing in density before colliding at the chamber centre. The magnetic field from the Helmholtz coil alters the magnetization of the iron core and thus the bias flux within the coaxial injectors, which is taken into account in the reported $\lambda_{\text {gun }}$ values.

We define the chamber coordinates in the poloidal plane by $(r, z)$, where the south pole is $(r=0 \mathrm{~cm}, z=-150 \mathrm{~cm})$ and the north pole is $(r=0 \mathrm{~cm}, z=+150 \mathrm{~cm})$. Diagnostics are placed at different toroidal angles $\phi$, and toroidal symmetry of the plasma structure is assumed for estimating plasma parameters. Experimental results suggest that this assumption may not always be valid. However, toroidal symmetry is not a requirement for accessing the parameter regimes for the physics studies of interest, nor for the studies themselves.

\subsection{Plasma injector}

Figure 2 illustrates the coaxial plasma injectors (Matsumoto et al. 2016a,b; Edo et al. 2018) that we use to create our plasmas. The inner radius of the outer electrode (anode) 
is $r_{\text {gun }}=4.15 \mathrm{~cm}$, leading to $\lambda_{\text {crit }} \approx 92 \mathrm{~m}^{-1}$ for spheromak formation. Thus, we desire $\lambda_{\text {gun }}>\lambda_{\text {crit }}$, by reducing $\psi_{\text {gun }}$, in order to create $\beta>1, \omega \tau>1$ magnetized plasmas.

The plasmas are created by (i) establishing a poloidal bias flux $\psi_{\text {gun }}$ between the coaxial electrodes, (ii) injecting neutral gas between the electrodes, (iii) preionizing the gas to create a plasma, (iv) accelerating the plasma by discharging current through the electrodes. To establish $\psi_{\text {gun }}$, an iron core surrounded by a copper winding is inserted into the inner coaxial electrode (cathode). A current $<3$ A (direct current) through the $4.1 \mathrm{~cm}$ diameter winding supplies $\psi_{\text {gun }}<0.4 \mathrm{mWb}$ within the $\sim 30 \mathrm{~cm}$ length region of plasma formation and acceleration in the coaxial injector. To vary $\lambda_{\text {gun }}$, we vary $\psi_{\text {gun }}$ and fix $I_{\text {gun }}$. Gas is injected by valves into the coaxial gun $3 \mathrm{~ms}$ before the trigger of the main electrode discharge.

In the present work, we use $\mathrm{H}$ and $\mathrm{He}$ gases for the south and north injectors, respectively. Helium is used for ion Doppler spectroscopy measurements. We only use $\mathrm{H}$ in the south injector, for which we observe better performance with $\mathrm{H}$ than with $\mathrm{He}$ (not yet understood). The south injector is on loan from TAE Technologies, Inc., and the north injector was modified, designed and built at WiPPL based on the TAE injector. The injected gas diffuses through a 'washer gun' (Fiksel et al. 1996), in which an applied preionization voltage $<1 \mathrm{kV}$ breaks down the gas, and a $1 \mathrm{kA}$ current sustains $100 \mathrm{~kW}$ of heating power. The washer guns use a $<1 \mathrm{kG}$ magnetic field produced with a small solenoid to assist in plasma breakdown. This field is roughly aligned with the poloidal magnetic field produced by the iron-core solenoid.

The main current $I_{\text {gun }}$ that accelerates the plasma out of the coaxial gun by the $\boldsymbol{J} \times \boldsymbol{B}$ force has a peak of $\approx 130 \mathrm{kA}$ and a risetime of $5 \mu \mathrm{s}$. The current is crowbarred to prevent ringing. For each injector, the main discharge circuit has a capacitance of $70 \mu \mathrm{F}$ operating at voltages of $10 \mathrm{kV}$, thereby storing $3.5 \mathrm{~kJ}$. Accounting for losses in the transmission line, the energy delivered to the injector is $\sim 0.5 \mathrm{~kJ}$, estimated from time-resolved current and voltage measurements. After the plasma is accelerated along the length of the electrodes, it travels $\sim 35 \mathrm{~cm}$ through the injector and drift tube before it enters the experimental chamber.

\subsection{Diagnostics}

\subsubsection{Visible fast-framing camera imaging}

We image the individual plasmas and plasma collisions using a fast-framing Phantom v710 camera with $1 \mu \mathrm{s}$ exposure times and $3 \mu \mathrm{s}$ interframe time. The camera is positioned at an approximately $45^{\circ}$ angle relative to the axial plasma-propagation direction. Narrow bandpass filters selectively admit $\mathrm{H}_{\alpha}, \mathrm{H}_{\beta}$, He-I and He-II plasma line emission. The imaging helps verify magnetic-signal timings and with experimental troubleshooting.

\subsubsection{Langmuir probe}

Electron densities and electron temperatures are measured using a multitip Langmuir probe. The 16 tips each have independent bias voltages to sample the current-voltage $(I-V)$ traces with $0.2 \mu$ s sampling period. The position of the Langmuir probe within the chamber can be adjusted, but for the data presented in this work, the probe was fixed at the chamber coordinates $(r=25 \mathrm{~cm}, z=10 \mathrm{~cm})$. The $n_{e}$ is given by (Cherrington 1982)

$$
n_{e}=\frac{I_{\mathrm{sat}}}{0.61 A_{p} e}\left(\frac{\mu m_{p}}{k_{B} T_{e}}\right)^{1 / 2},
$$

where $I_{\text {sat }}$ is the ion saturation current, $A_{p}$ is the probe area, $e$ is the elementary charge and $m_{p}$ is the proton mass. We use $\mu=1,4$ and 2.5 for $\mathrm{H}$, He and merged $\mathrm{H}-\mathrm{He}$ plasmas, 
respectively. The $T_{e}$ is inferred by fitting

$$
I=I_{\text {sat }}\left\{\exp \left[\frac{e\left(V-V_{f}\right)}{k_{B} T_{e}}\right]-1\right\}
$$

to the exponential part of the $I-V$ curve.

The discrete nature of sampling with a multitip probe introduces slightly more error than a typical swept Langmuir probe. All error bars presented here represent statistical uncertainties introduced via measurement and propagated in the analysis, but do not represent the typically much larger errors from Langmuir probe theory (Hutchinson 2002). Looking at the quiescent period after the plasmas have collided, we estimate the standard deviations in the measured $I$ and $V$ to be $0.2 \mathrm{~V}$ and $1 \mathrm{~mA}$, respectively. Using these values, we take the $I-V$ data and generate an $N=10^{4}$ size population of mock data with standard deviations around those values. By repeating these calculations at different times and for different shots, we find a $4 \%$ uncertainty in $T_{e}$ and a $5 \%$ uncertainty in $n_{e}$, which are the combination of the (non-systematic) uncertainties in measurement and the fitting routine. These represent lower bounds in the error, as they only include noise and not theoretical or systematic error.

\subsubsection{Magnetic probe arrays}

The magnetic fields and velocities are measured using an array of Bdot probes ('hook magnetic probe' in figure 3), where the probe loop voltage $V \propto \partial B / \partial t$. The Bdot probe array consists of 11 locations equally spaced between $(r=16 \mathrm{~cm}, z=-20 \mathrm{~cm})$ and $(r=$ $16 \mathrm{~cm}, z=40 \mathrm{~cm}$ ) for the data presented in this work. At each array location there are six loops - three orthogonal pairs of oppositely wound loops - to measure the three spatial components of the magnetic field and minimize common mode noise. Data are recorded at a $0.1 \mu \mathrm{s}$ sampling rate and numerically integrated to provide values of the magnetic field. The Bdot-coil areas are calibrated to within a few percent. There is a systematic uncertainty of the entire probe array of $\sim 1 \mathrm{~cm}$.

There are two other Bdot-probe arrays in the chamber ('axial magnetic probe' and 'radial magnetic probe' in figure 3), which provide magnetic signals at different radii and toroidal positions. These arrays help determine the radial extent of the plasma $(\sim 30 \mathrm{~cm}$ radius) as a function of toroidal angle. We also observe $\sim 30 \mathrm{~km} \mathrm{~s}^{-1}$ plasma radial expansion, which affects the ion Doppler spectroscopy data.

\subsubsection{Ion Doppler spectroscopy}

Ion temperatures are measured using ion Doppler spectroscopy. We observe Doppler broadening of the $468.6 \mathrm{~nm}$ He-II line along a radial viewing chord at $z=10 \mathrm{~cm}$. Light is collected using a $2.54 \mathrm{~cm}$ collimator, a $10 \mathrm{~nm}$ bandwidth monochrometer, $3 \mu \mathrm{m}$ fibre bundles and fed into a Czerny-Turner type spectrometer, as described in Den Hartog \& Fonck (1994). We obtain measurements at a sampling rate of $\sim 1 \mu \mathrm{s}$. The measured broadening is a convolution of broadening from the following sources: the ion temperature, the spectrometer instrumental broadening and the plasma radial-expansion velocity. The result is $\sigma_{\text {measured }}^{2}=\sigma_{\mathrm{Ti}}^{2}+\sigma_{\text {instr }}^{2}+\sigma_{\text {exp }}^{2}$, where $\sigma$ is the Gaussian broadening, i.e. full-width at half-maximum (known as FWHM). The instrumental broadening at $T_{i}=30 \mathrm{eV}$ is equivalent to $\sigma_{\text {instr }}=1.5 \mathrm{eV}$, but the relative contribution increases for smaller $T_{i}$. Broadening from the $\sim 30 \mathrm{~km} \mathrm{~s}^{-1}$ plasma radial expansion is equivalent to $\sigma_{\text {exp }} \sim 9 \mathrm{eV}$. As $T_{e}$ cools below $\sim 10 \mathrm{eV}$, the emission drops rapidly, and $T_{i}$ cannot be measured. 


\section{Experimental results}

\subsection{Estimated plasma parameters}

The nature of head-on merging of two plasmas, e.g. whether they interpenetrate or form sonic or MHD shocks, depends on the plasma parameters just prior to merging. As the plasmas propagate with axial speed $v \sim 70 \mathrm{~km} \mathrm{~s}^{-1}$ into the chamber from the magnetized coaxial guns, they expand into a $50 \mathrm{G}$ applied background magnetic field that is oriented along the propagation direction ( $z$ axis). The plasmas expand radially (with instantaneous radius $R$ ) and axially (with instantaneous length $L$ ) each by a factor of $\sim 8$ from the initial size near the gun to the final size near the chamber centre, with volume $V \propto L R^{2}$. Near the chamber centre, we measure the plasma $R, L, n_{e}, T_{e}, T_{i}, B$ and $v$. In the drift tube near the gun, we measure $B$ during separate experiments, and we infer the other quantities by making assumptions about the expansion scalings (i.e. conservation of particles and resistive diffusion of magnetic flux), and verify the consistency of the inferred parameters near the gun with that of prior work using similar plasma injectors (Matsumoto et al. 2016a,b; Edo et al. 2018).

Making the assumptions of conservation of particle and exponentially decaying magnetic flux while the expanding plasma propagates from the gun to the chamber centre, we have the following relations: $n \propto L^{-1} R^{-2} ; B_{r}, B_{\phi} \propto L^{-1} R^{-1} \mathrm{e}^{-C t}$; and $B_{z} \propto R^{-2} \mathrm{e}^{-C t}$ with propagation time $t$ and a constant $C$. While it is tempting to assume adiabatic expansion $\left(P V^{\gamma}=\right.$ constant, with $\left.\gamma=5 / 3\right)$ to infer the dependence of $T$ on plasma volume $V$, the strict adiabatic-cooling rate $T \propto L^{-2 / 3} r^{-4 / 3}$ would lead to unrealistic $>\mathrm{keV}$ temperatures near the gun when extrapolated from measured temperatures near the chamber centre. Instead, as a lower bound, we take $T_{i} \approx$ constant. At higher densities near the gun, $T_{e}$ and $T_{i}$ equilibrate over $\sim 1 \mu \mathrm{s}$ time scales, but at the lower densities near the chamber centre, the ion-electron equilibration time is longer than the $\sim 20 \mu$ s propagation time. Thus, $T_{e}$ decreases from the initial $T_{e} \approx T_{i}$ near the gun to the measured $T_{e}<T_{i}$ near the chamber centre, due to radiation and $P \mathrm{~d} V$ work on the background magnetic field. Concurrently, resistive dissipation of the magnetic field causes Ohmic heating of the electrons. To put bounds on parameters rather than to be predictive of the precise plasma temperature between the gun and the chamber centre, we simply model a linear decrease in $T_{e}$ during propagation.

Based on the relations and information discussed above, we provide estimates of parameters near the gun and near the chamber centre in table 1, which suggests that thermal pressure is greater than magnetic pressure (i.e. $\beta>1$ ) both near the gun and near the chamber centre. While the radial expansion speed measured by the B-dot probe array near the chamber centre is $\sim 30 \mathrm{~km} \mathrm{~s}^{-1}$, the average expansion speed calculated during propagation through the chamber (from figure 4) is $\sim 13 \mathrm{~km} \mathrm{~s}^{-1}$. Both observations of the radial expansion speed are less than the ion sound speed $C_{s}$ or Alfvén speed $V_{A}$ near the gun (in table 1). Near the chamber centre, the measured expansion speed is still subsonic. During head-on merging, the plasmas are super-Alfvénic and slightly supersonic, and thus it may be expected that MHD shocks could form. However, during initial head-on merging of two plasmas, the classical ion-ion Coulomb interpenetration length between the merging plasmas is an appreciable fraction of $L$, and thus it may be expected that any shock formation will be delayed if it forms at all, as observed in prior work with merging supersonic plasmas (Moser \& Hsu 2015; Langendorf et al. 2019). By interpolating the data in table 1 , we show in figure 4 the time evolution of parameters for an individual plasma as it propagates to the centre of the chamber before merging. Notably, $\beta, \omega_{i} \tau_{i}$ and $\omega_{e} \tau_{e}$ are anticipated to be equal to or greater than unity for an individual plasma during propagation 
Parameters for Individual Plasmas During Propagation in Chamber
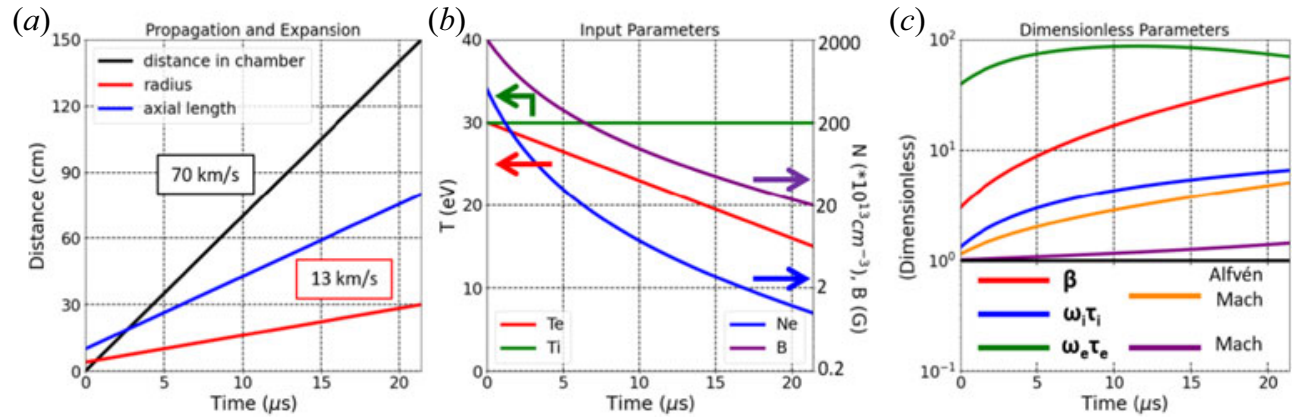

FIGURE 4. Anticipated plasma parameters versus time for individual plasmas as they propagate to the centre of the chamber. Values are based on interpolating the data in table 1 and serve to place bounds on parameters rather than to precisely predict behaviour during propagation.

and expansion into the chamber. A key question is whether this is also true upon merging, where densities can be much higher than for an individual plasma.

\subsection{Negligible effect of neutrals}

In this subsection, we show that the effect of neutrals should be negligible.

First, we consider ion-neutral collisionality (dominated by charge exchange) within the plasmas before merging. The plasmas generated from the coaxial injectors are highly ionized. We utilize the PrismSpect atomic modelling software (MacFarlane et al. 2004) with the temperature and density conditions in table 1 to estimate the fraction of neutral particles to be $<3 \times 10^{-6}$ near the gun and $<2 \times 10^{-6}$ near the chamber centre. Within each plasma, the ion-neutral collision time is $\tau_{C X}=v_{C X}^{-1} \approx\left(n_{n} \sigma_{C X} v_{i, n}\right)^{-1}$, where we assume that the relative speed between ions $\left(T_{i}=30 \mathrm{eV}\right)$ and cold neutrals is $v_{i, n}=54 \mathrm{~km} \mathrm{~s}^{-1}$. Based on the high ionization fraction, the neutral density is $n_{n}<1.5 \times$ $10^{10} \mathrm{~cm}^{-3}$. The $\mathrm{H}^{1+}-\mathrm{H}^{0}$ charge exchange cross-section is $\sigma_{C X} \sim 3 \times 10^{-15} \mathrm{~cm}^{2}$ (Smirnov 2000). Therefore, we estimate that $\tau_{C X} \approx 4 \mathrm{~ms}$, much larger than the experimental duration. Comparatively, the thermal ion-ion collision times $\tau_{i}$ are $\sim 68$ ns near the gun and $\sim 34 \mu$ s near the chamber centre. Thus, ion-neutral collisionality within the plasma is a small effect.

Next we consider the collisionality between the plasma ions and the background neutrals in the vacuum chamber. We expect plasmas injected into the chamber $\left(\sim 70 \mathrm{~km} \mathrm{~s}^{-1}\right)$ to run ahead of injected neutrals (at room temperature $0.026 \mathrm{eV}$, thermal velocity $v_{\mathrm{th}, n}<$ $1.5 \mathrm{~km} \mathrm{~s}^{-1}$ ). The vacuum chamber base pressure $\sim 4 \mu$ Torr corresponds to a neutral background density of $n_{n} \approx 1.3 \times 10^{11} \mathrm{~cm}^{-3}$. The collision time for plasma ions colliding with the stationary background neutrals is $\tau_{\text {background }}=v_{\text {background }}^{-1} \approx\left(n_{n} \sigma_{C X} v_{i}\right)^{-1}$, where $v_{i} \sim 70 \mathrm{~km} \mathrm{~s}^{-1}$ is the ion speed. We calculate this collision time to be $\tau_{\text {background }} \approx 400 \mu \mathrm{s}$, again larger than the experimental duration. Comparatively, the ion-ion collision time for the two counter-streaming merging plasmas is approximately equal to the ion-ion slowing time $\tau_{i i, s}=v_{i i, s}^{-1} \approx\left(v_{\text {rel }} / 4 L_{i i, s}\right)^{-1}$ (Messer et al. 2013; Merritt et al. 2014), where $v_{\text {rel }}=2 v_{i} \sim 140 \mathrm{~km} \mathrm{~s}^{-1}$ is the relative plasma velocity and $L_{i i, s} \approx 57 \mathrm{~cm}$ is the ion-ion slowing length, which gives $\tau_{i i, s} \approx 16 \mu \mathrm{s}$. This calculation is a characteristic slowing time for $\mathrm{H}-\mathrm{H}$ merging; for $\mathrm{H}-\mathrm{He}$ and $\mathrm{He}-\mathrm{H}$ the calculated slowing times are $3 \mu \mathrm{s}$ and $27 \mu \mathrm{s}$, respectively. Thus, the effect of neutral collisionality is also negligible in the merging between opposing plasmas. 
Parameter

Propagation distance $(\mathrm{cm})$

Time $t$ ( $\mu s)$

Plasma radius $R(\mathrm{~cm})$

Plasma length $L(\mathrm{~cm})$

Electron density $n_{e}\left(\mathrm{~cm}^{-3}\right)$

Electron temperature $T_{e}(\mathrm{eV})$

Ion temperature $T_{i}(\mathrm{eV})$

Magnetic field $B(\mathrm{G})$

Thermal electron collision time $\tau_{e}$

Thermal ion collision time $\tau_{i}$

$\beta$

$\omega_{i} \tau_{i}$

$\omega_{e} \tau_{e}$

Axial plasma speed $v\left(\mathrm{~km} \mathrm{~s}^{-1}\right)$

Sound speed $C_{S}\left(\mathrm{~km} \mathrm{~s}^{-1}\right)$

Alfvén speed $V_{A}\left(\mathrm{~km} \mathrm{~s}^{-1}\right)$

Mach number $M$

Alfvén Mach number $M_{A}$

Electron thermal mean free path $\lambda_{\mathrm{mfp}, e}(\mathrm{~cm})$

Ion thermal mean free path $\lambda_{\mathrm{mfp}, i}(\mathrm{~cm})$

Ion-ion interpenetration length for merging plasmas $L_{i i, s}(\mathrm{~cm})$

Ion gyroradius $\rho_{i}(\mathrm{~cm})$

Ion inertial length $c / \omega_{p i}(\mathrm{~cm})$
Near gun Near chamber centre

\begin{tabular}{|c|c|}
\hline 0 & 150 \\
\hline 0 & 20 \\
\hline 4 (est.) & 30 (meas.) \\
\hline 10 (est.) & 80 (meas.) \\
\hline $5 \times 10^{15}$ (est.) & $10^{13}$ (meas.) \\
\hline 30 (est.) & 15 (meas.) \\
\hline 30 (est.) & 30 (meas.) \\
\hline 2000 & 20 (meas.) \\
\hline $1 \mathrm{~ns}$ & $0.2 \mu \mathrm{s}$ \\
\hline $68 \mathrm{~ns}$ & $34 \mu \mathrm{s}$ \\
\hline 3 & 45 \\
\hline 1 & 7 \\
\hline 40 & 70 \\
\hline 70 (est.) & 70 (meas.) \\
\hline 70 & 50 \\
\hline 60 & 14 \\
\hline 1 & 1.4 \\
\hline 1.2 & 5 \\
\hline 0.3 & 30 \\
\hline 0.4 & 180 \\
\hline (N/A) & 57 \\
\hline 0.3 & 30 \\
\hline 0.3 & 7 \\
\hline
\end{tabular}

TABle 1. Measured (meas.) and estimated (est.) values of plasma parameters (for single plasmas) for the experiments reported in this paper, assuming $Z=1, \mu=1, \gamma=5 / 3$ and $\ln \Lambda=10$.

\subsection{Measurements near the chamber centre}

The primary results of this work are the measurement of plasma parameters - density, temperature, and magnetic field - from both individual plasmas and merged plasmas via head-on collisions, which occur near the $(r<30 \mathrm{~cm}, z=10 \mathrm{~cm})$ chamber position. We show evidence for obtaining transiently the desired state of $\beta>1$ and $\omega_{i} \tau_{i}>1$ for both situations. Figure $5(a, b)$ shows visible imaging of individual plasmas coming from the south and north sides of the chamber, respectively, and also of a head-on plasma collision (panel $(c)$ ) between the two individual plasmas. We use the emission seen on the sides of the probes to observe and verify from which direction the plasma is coming. Estimates of Bohm losses to probe surfaces are small compared with total particle inventories, and thus we expect the probes to not significantly perturb the plasma.

Here, the south $\mathrm{H}$ plasma has $\lambda_{\text {gun }, S}=600 \mathrm{~m}^{-1}$, and the north $\mathrm{He}$ plasma has $\lambda_{\text {gun }, N}=$ $1200 \mathrm{~m}^{-1}$. Depending upon the formation and propagation dynamics, the ratio of toroidal to poloidal magnetic fields and corresponding $\lambda_{\text {plasma }}$ for the plasma can be different from $\lambda_{\text {gun }}$, but $\lambda_{\text {gun }}$ influences the initial plasma state. Since $\lambda_{\text {gun }}$ satisfies $\infty>\lambda_{\text {gun }}>\lambda_{\text {crit }}=$ $92 \mathrm{~m}^{-1}$, we are in the desired parameter regime for obtaining $\beta>1$ and $\omega_{i} \tau_{i}>1$ plasmas.

Magnetic-field data are obtained by integrating the Bdot-probe-array signals. Figure 6 shows the spatio-temporal evolution of the magnetic-field components for an individual $\mathrm{H}$ plasma from the south (shot 46330), an individual He plasma from the north (shot 46338) 


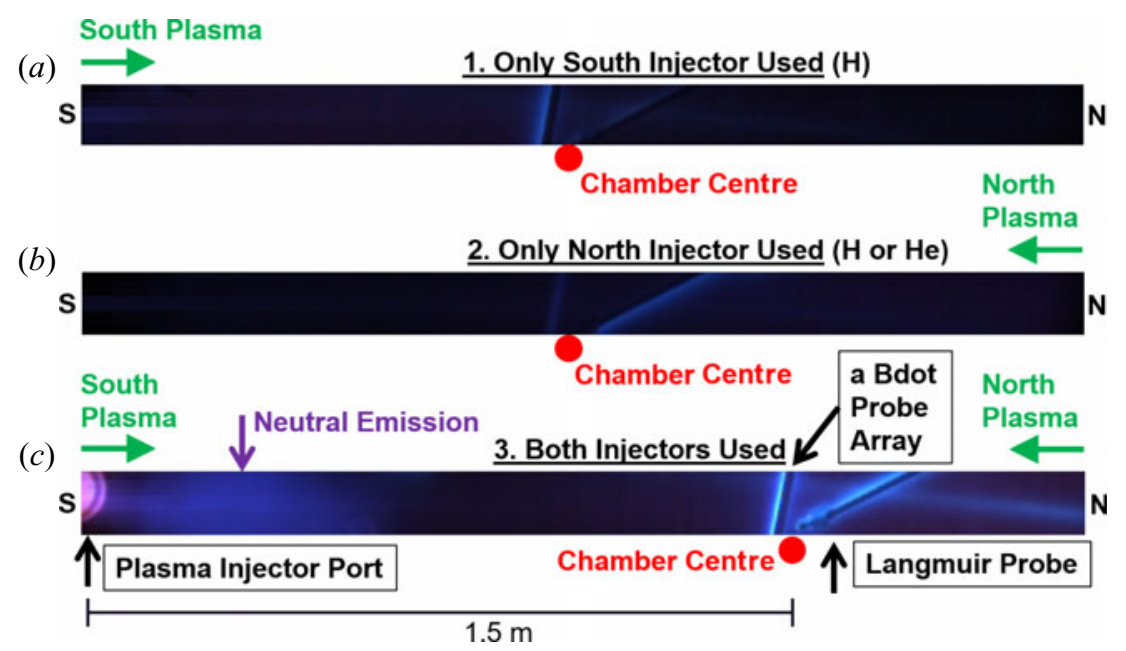

FIGURE 5. Fast-framing camera images of visible self-emission for plasmas from $(a)$ south injector only, $(b)$ north injector only, and $(c)$ both injectors. The main purpose of these images is to verify which sides of the probes light up due to the incoming plasma(s).

and for an $\mathrm{H}-\mathrm{He}$ collision (shot 46343). From left to right, the three columns of coloured contour plots are for the radial $B_{r}$, toroidal $B_{\phi}$ and axial $B_{z}$ fields $(z=0$ corresponds to the chamber centre). For the contour plots, the Bdot-probe array is oriented along $z$ and positioned at $r=16 \mathrm{~cm}$. For reference, the arrival of the density signal can be seen in figure 7.

Based on the slopes of the magnetic contours, we infer individual plasma axial velocities of $\sim 70 \mathrm{~km} \mathrm{~s}^{-1}$. As the plasmas propagate through the chamber and radially expand, they advect the $50 \mathrm{G}$ background axial magnetic field in the radial direction, reducing the magnitude of the $B_{z}$ component within the plasma radius. In the $\mathrm{H}-\mathrm{He}$ collision case, we observe larger magnetic-field strengths of the non-axial components ( $\sim 30 \mathrm{G}$ compared with $<20 \mathrm{G}$ ), and the axial field is reduced to $B_{z}<20 \mathrm{G}$ for a longer duration. Due to stagnation of the colliding plasmas, the magnetic signals last for $>40 \mu$ s near the chamber centre for the $\mathrm{H}-\mathrm{He}$ collision case compared with $\sim 20 \mu \mathrm{s}$ for the individual plasmas that propagate past the chamber centre. In future work, we intend to better characterize the anticipated diamagnetic effects based on the relative polarities of the background field compared with the magnetized plasmas.

Figure 7 shows the time evolution of $T_{e}$ and $n_{e}($ at $r=25 \mathrm{~cm}, z=10 \mathrm{~cm})$ and total $B$ (at $r=16 \mathrm{~cm}, z=10 \mathrm{~cm}$, from figure 6) for an individual $\mathrm{H}$ plasma from the south (shot 46330), an individual He plasma from the north (shot 46338) and for an $\mathrm{H}-\mathrm{He}$ collision (shot 46343). For shots 46338 and 46343, for which there is He present, $T_{i}$ is also plotted. Compared with the plasma from the north, the south plasma density persists for less time $(<20 \mu \mathrm{s})$ and causes less reduction of the background magnetic field. The $\mathrm{H}-\mathrm{He}$ collision case has a peak density above the value for the two individual plasmas, and at $>60 \mu \mathrm{s}$, the magnetic signals decrease to $<15 \mathrm{G}$, although density is still present. Additionally, $T_{e}$ for both the individual and colliding plasmas are $T_{e} \approx 15 \mathrm{eV}$. The $T_{i}$ data show that $T_{i}>30 \mathrm{eV}>T_{e}$. At $>60 \mu \mathrm{s}$, the $T_{i}$ signal falls to zero for low density and low electron temperature.

Figure 8 shows the derived values of $\beta$ and $\omega_{i} \tau_{i}$ versus $t$, based on the quantities in figure 7. Because experiments with the individual plasma from the south (shot 46330) 


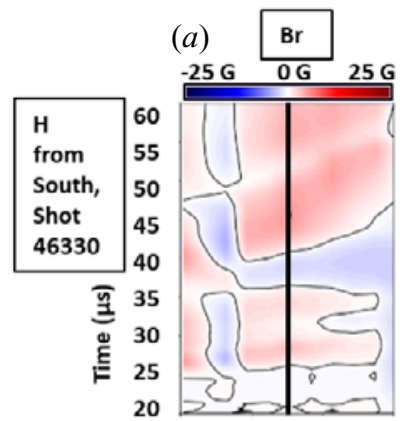

(e)

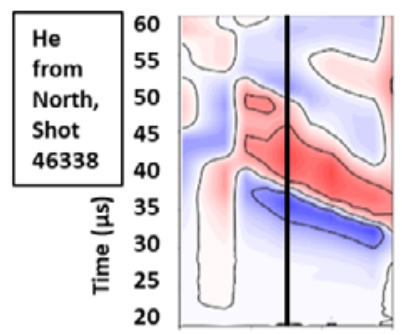

(i)

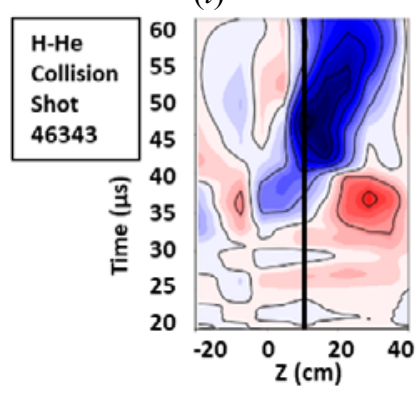

(b)

$-25 \mathrm{G} \quad 0 \mathrm{G} \quad 25 \mathrm{G}$

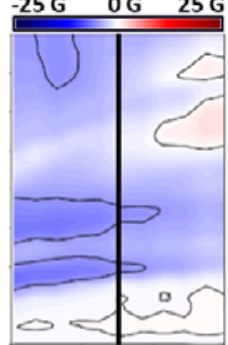

$(f)$

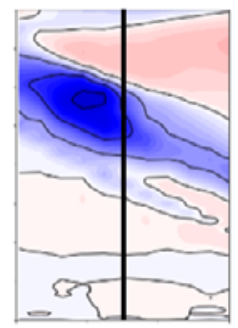

$(j)$

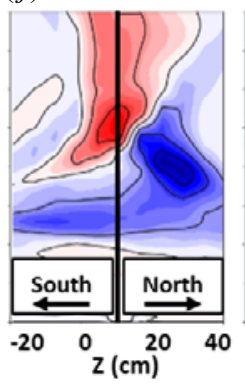

(c)

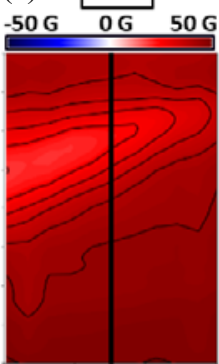

$(g)$

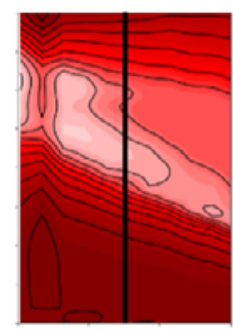

$(k)$

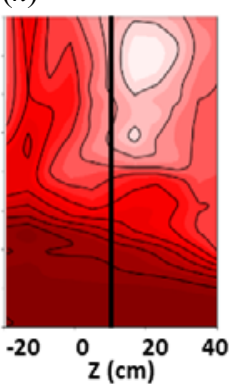

(d) Magnetic Fields at $\mathrm{z}=10 \mathrm{~cm}$
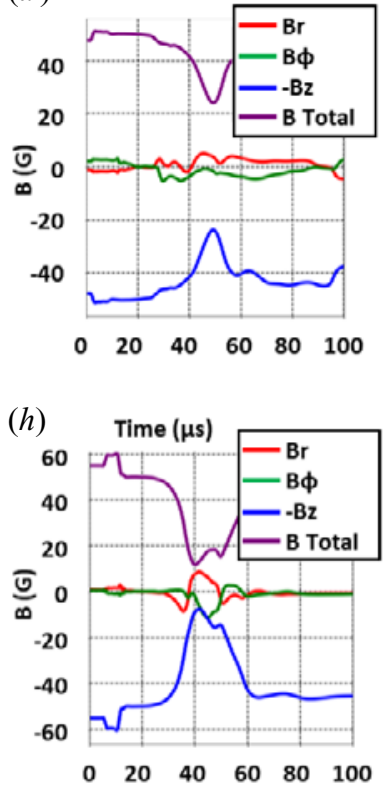

$(l)$

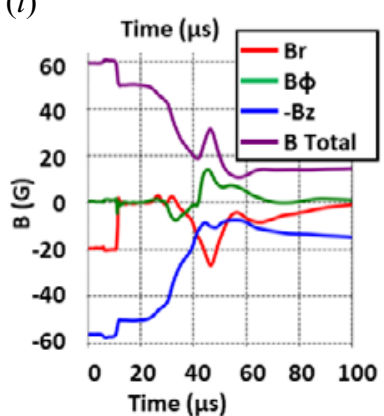

FIGURE 6. $(a-c, e-g, i-k)$ Magnetic-field components versus $z$ (at $r=16 \mathrm{~cm}$ ) and $t$ for $(a-d)$ an individual $\mathrm{H}$ plasma, $(e-h)$ an individual He plasma and $(i-l)$ an $\mathrm{H}-\mathrm{He}$ collision. A signal travelling up and to the right is travelling through the chamber from south to north. The contour lines represent $5 \mathrm{G}$ increments. $(d, h, l)$ Lineouts (corresponding to the vertical black lines in the contour plots) of magnetic-field components and total $B=\left(B_{r}^{2}+B_{\phi}^{2}+B_{z}^{2}\right)^{1 / 2}$ versus $t$ at $z \approx 10 \mathrm{~cm}$ (including the applied $B_{z}$ from the Helmholtz coil). To avoid obscuring the $B$-total trace, $-B_{z}$ is plotted.

uses only $\mathrm{H}$ gas, we did not obtain direct $T_{i}$ measurements and therefore assume $T_{i}=T_{e}$ for those shots (which serves as a lower bound for $T_{i}, \beta$ and $\omega_{i} \tau_{i}$ ). In figure $8(b, c)$, the red and blue traces (where $T_{i}=T_{e}$ is assumed) can be compared with the green and purple traces (based on measured $T_{i}$ ). We observe $\beta>1$ and $\omega_{i} \tau_{i}>1$ for $>20 \mu \mathrm{s}$ in both the individual $\mathrm{H}$ and $\mathrm{He}$ plasmas and in the $\mathrm{H}-\mathrm{He}$ collision. In particular, at the $50 \mu \mathrm{s}$ time of peak density in the collision, we obtain $\beta \approx 100$ and $\omega_{i} \tau_{i} \approx 4$ (using the measured $T_{i}$ values). Compared with the individual-plasma cases, which also obtain the desired $\beta, \omega \tau>1$ regime due to plasma expansion, the collision case gives higher densities and durations which expands the parameter regime available for study, and is more relevant for scaling to regimes of interest for an MIF target. 

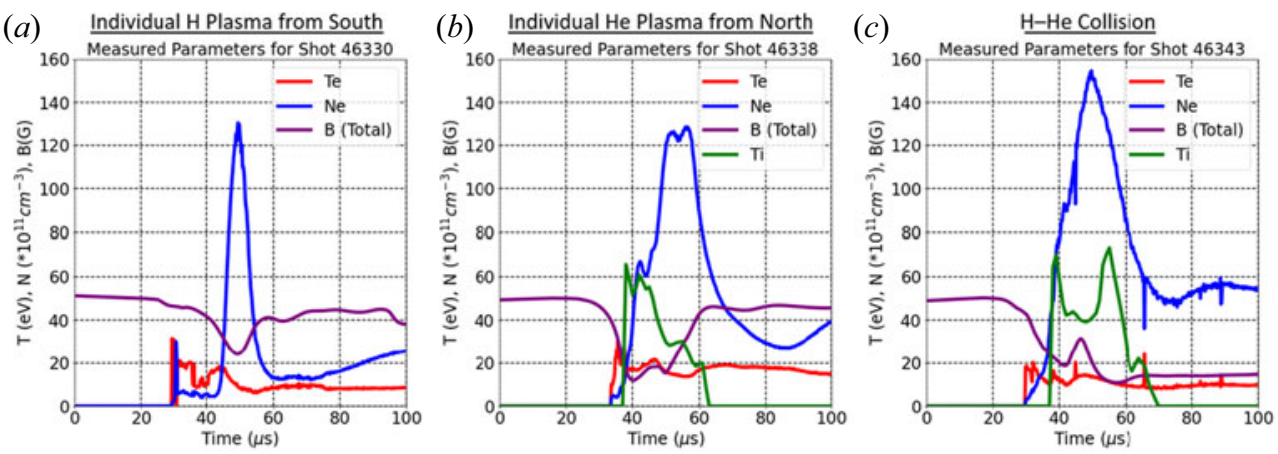

Figure 7. Plots of $T_{e}$ and $n_{e}$ (at $z=10 \mathrm{~cm}, r=25 \mathrm{~cm}$ ), total $B$ (at $z=10 \mathrm{~cm}, r=16 \mathrm{~cm}$, from figure 6) and $T_{i}$ (when He is present; radial viewing chord at $z=10 \mathrm{~cm}$ ) versus $t$ for an (a) individual $\mathrm{H}$ plasma, (b) individual He plasma and (c) $\mathrm{H}-\mathrm{He}$ collision.
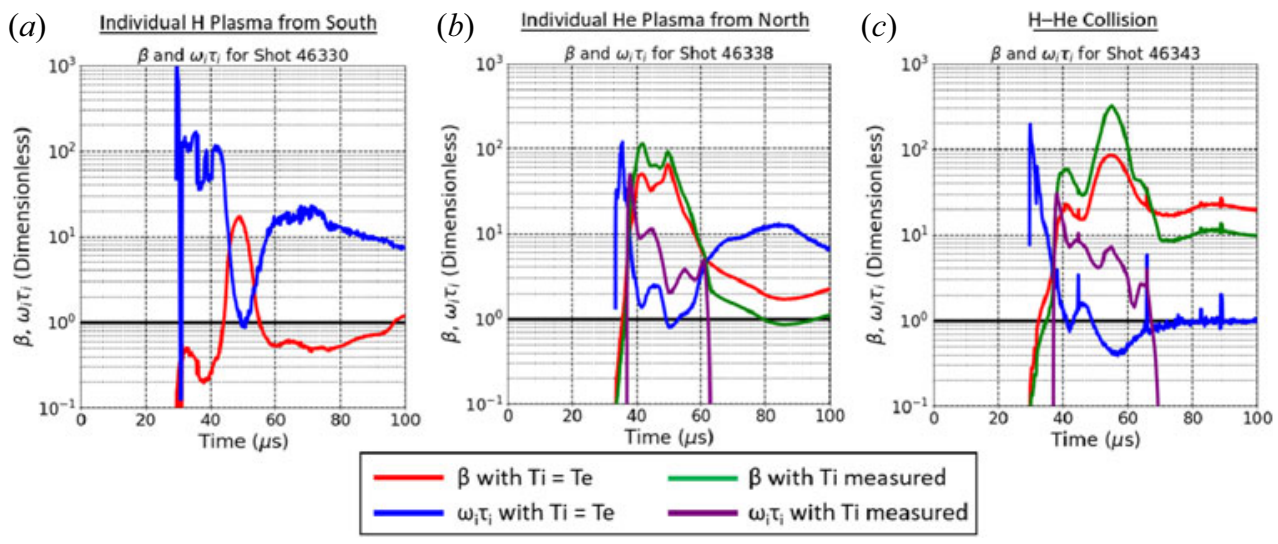

FIGURE 8. Derived values of $\beta$ and $\omega_{i} \tau_{i}$ versus $t$, based on the quantities from figure 7 , showing that there are time windows up to $20 \mu \mathrm{s}$ in duration during which $\beta$ and $\omega_{i} \tau_{i}$ are simultaneously greater than unity, for all three cases of $(a)$ individual $\mathrm{H}$ plasma, $(b)$ individual He plasma and (c) $\mathrm{H}-\mathrm{He}$ collision.

We find that the plasma from the north injector has larger $B_{r}$ and $B_{\phi}$ than the plasma from the south injector. The $50 \mathrm{G}$ background $B_{z}$ from the Helmholtz coil is used to prevent radial expansion of the plasmas. Without the background field, we observe a significant decrease to near zero in the magnetic field signal in the plasma from the south injector, but we do still observe (smaller) magnetic signals in the plasma from the north injector. Studying the plasma collision without the background magnetic field is useful to support the future goals of studying collisions of more than two plasmas. In a possible future case of colliding six plasmas together (e.g. three orthogonal sets of head-on collisions), a Helmholtz-coil magnetic field would not be able to align with the propagation direction for six plasmas.

\section{Discussion and future work}

We intend to build on this work in upcoming experimental campaigns by (i) exploring the time dynamics for the plasma collisions, especially looking into how the energy is distributed within the system, (ii) obtaining a spatial map of plasma parameters by 
scanning probe positions within the chamber, and (iii) performing a greater number of shots per position to obtain statistics on density, temperature and magnetic-field fluctuations. During the experimental campaigns reported here, we did not complete a probe spatial scan because the individual plasmas did not form as reproducibly as desired. A likely reason for the unrepeatable nature is preionization that is not toroidally symmetric within the coaxial injector. Future work will test more-uniform gas injection and preionization systems. Preliminary engineering improvements using a new more-uniform annular preionization system, a reduced gas injection timing delay from $3 \mathrm{~ms}$ to $2 \mathrm{~ms}$, and four gas injectors instead of one around the annular gun nozzle have shown promise in creating more-reproducible plasmas with $\sim 95 \%$ reliability for obtaining similar plasma parameters shot-to-shot. Equivalent levels of engineering improvements to plasma guns and their subsystems have recently been demonstrated in Yates et al. (2020), which reported significantly improved balance of plasma mass injection in six- and seven-gun experiments as compared with Hsu et al. (2018). Continuation of these experiments on the BRB will focus on better characterization of and generation of small-scale, tangled fields in these merged, $\beta>1, \omega \tau>1$ plasmas, as well as the exploration and variation of additional parameters, such as radiative cooling, magnetic Prandtl number, etc., of relevance to fundamental plasma physics and plasma-astrophysics questions.

Future studies of processes related to astrophysical phenomena including MHD turbulence and magnetic dynamo will depend upon the plasma characteristics. Required resolution of spatial scales and time scales for MHD turbulence depend upon the Alfvén speed. From the approximate parameters obtained in the present work (see table 1), a 50 $\mathrm{cm}$-scale plasma environment should last $>35 \mu$ s to support at least one crossing time associated with a $\sim 14 \mathrm{~km} \mathrm{~s}^{-1}$ Alfvén speed. The plasma needs to support $\sim 10$ ion inertial lengths $(0.3-7 \mathrm{~cm})$ and ion gyroradii $(0.3-30 \mathrm{~cm})$ to allow turbulent cascades to develop. These turbulent cascades will be experimentally characterized while covering over two orders of magnitude in spatial scale by conducting millimetre-scale measurements. For studies of the magnetic dynamo, we plan to obtain large-enough ( $\gg 1)$ magnetic Prandtl numbers (the ratio of magnetic Reynolds number to Reynolds number, $\operatorname{Pr}=R_{m} / \operatorname{Re} \propto T^{4}$ ) and support at least one global turnover time over which the $50 \mathrm{~cm}$-scale plasma can be advected by $\sim 50 \mathrm{~km} \mathrm{~s}^{-1}$ flows, in $\sim 10 \mu \mathrm{s}$.

Meanwhile, upcoming experiments on the Plasma Liner Experiment (known as the PLX) (Hsu et al. 2018; Yates et al. 2020) at Los Alamos National Laboratory will explore the merging of six or more plasmas to create $\beta>1, \omega \tau>1$ plasmas, at higher densities and temperatures than on the $\mathrm{BRB}$, as a potential MIF target. To advance this MIF concept, future work on the BRB will explore formation of tangled magnetic fields within $\beta>1, \omega \tau>1$ plasmas, for which the magnetic field connection length is much longer than the local magnetic field scale size. These studies will evaluate thermal transport properties compared with theoretical predictions for diffusion coefficients in plasmas with tangled magnetic fields (Kadomtsev \& Pogutse 1978; Rechester \& Rosenbluth 1978; Chandran \& Cowley 1998).

\section{Conclusions}

In this work, we successfully used coaxial plasma-gun injectors to study individual and head-on-merging plasmas on the BRB at the WiPPL. By tuning the injector parameter $\lambda_{\text {gun }}=\mu_{0} I_{\text {gun }} / \psi_{\text {gun }}$ within an intermediate range between $\lambda_{\text {crit }}<\lambda_{\text {gun }}<\infty$, we were able to demonstrate the formation of a transient plasma with both $\beta>1$ and $\omega_{i} \tau_{i}>1$. This is a promising first step toward establishing a new experimental platform for studying frontier, weakly magnetized, high- $\beta$ plasma physics, e.g. the topics described in $\S 5$. Compared with individual plasmas, the head-on collisions (i) increase the duration for which the 
desired plasma state exists at a particular location and (ii) increase the magnitudes of $n, B_{r}$, and $B_{\phi}$, which widens the parameter space compared with that achievable with individual plasmas.

\section{Acknowledgements}

T.B. and D.A.E. contributed equally to these experiments. The authors gratefully acknowledge J. Olson, J. Wallace, D. Den Hartog and the rest of the WiPPL team for their technical support. We acknowledge H. Gota, T. Roche, I. Allfrey and TAE Technologies for providing one of the plasma injectors and its design. Los Alamos National Laboratory (LANL) staff were supported by the LANL Laboratory Directed Research and Development (LDRD) program under DOE contract no. 89233218CNA000001. University of Wisconsin staff and the WiPPL user facility are supported by the DOE Office of Science, Fusion Energy Sciences under award no. DE-SC0018266. The BRB construction was supported by a National Science Foundation Major Research Instrumentation grant.

Editor Troy Carter thanks the referees for their advice in evaluating this article.

\section{Declaration of interests}

The authors report no conflict of interest.

\section{REFERENCES}

Balbus, S. A. \& Hawley, J. F. 1998 Instability, turbulence, and enhanced transport in accretion disks. Rev. Mod. Phys. 70, 1.

Bellan, P. M. 2000 Spheromaks. Imperial College Press.

Bruno, R. \& CARbone, V. 2013 The solar wind as a turbulence laboratory. Living Rev. Solar Phys. 2, 2.

Chandran, B. D. G. \& Cowley, S. C. 1998 Thermal conduction in a tangled magnetic field. Phys. Rev. Lett. 80, 3077.

Cherrington, B. E. 1982 The use of electrostatic probes for plasma diagnostics - a review. Plasma Chem. Plasma Process. 2 (2), 113-140.

Cooper, C. M., Wallace, J., Brookhart, M., Clark, M., Collins, C., Ding, W. X., Flanagan, K., Khalzov, I., Li, Y., Milhone, J., et al. 2014 The madison plasma dynamo experiment: a facility for studying laboratory plasma astrophysics. Phys. Plasmas 21 (1), 013505.

Cothran, C. D., Falk, A., Fefferman, A., Landreman, M., Brown, M. R. \& Schaffer, M. J. 2003 Spheromak merging and field reversed configuration formation at the Swarthmore Spheromak Experiment. Phys. Plasmas 10, 1748.

Den HARTOG, D. J. \& FonCK, R. J. 1994 A fast spectroscopic diagnostic for the measurement of plasma impurity ion dynamics. Rev. Sci. Instrum. 65, 3238.

Edo, T., Asai, T., Tanaka, F., Yamada, S., Hosozawa, A., Kaminou, Y., Gota, H., Roche, T., AlLfREY, I., OSIN, D., et al. 2018 Performance improvement of a magnetized coaxial plasma gun by adopting iron-core bias coil and pre-ionization systems. Plasma Fusion Res. 13, 3405062.

Fiksel, G., Almagri, A. F., Craig, D., Iida, M., Prager, S. C. \& SARFF, J. S. 1996 High current plasma electron emitter. Plasma Sour. Sci. Technol. 5 (1), 78-83.

Forest, C. B., Flanagan, K., Brookhart, M., Clark, M., Cooper, C. M., Désangles, V., Egedal, J., EndRizZI, D., Khalzov, I. V., LI, H., et al. 2015 The wisconsin plasma astrophysics laboratory. J. Plasma Phys. 81, 345810501.

Gomez, M. R., Slutz, S. A., Sefkow, A. B., Sinars, D. B., Hahn, K. D., Hansen, S. B., Harding, E. C., Knapp, P. F., Schmit, P. F., Jennings, C. A., et al. 2014 Experimental demonstration of fusion-relevant conditions in magnetized liner inertial fusion. Phys. Rev. Lett. 113, 155003.

Guo, H. Y., Binderbauer, M. W., Barnes, D., Putvinski, S., Rostoker, N., Sevier, L., Tuszewski, M., Anderson, M. G., Andow, R., Bonelli, L., et al. 2011 Formation of a 
long-lived hot field reversed configuration by dynamically merging two colliding high- $\beta$ compact toroids. Phys. Plasmas 18, 056110.

Hsu, S. C., Awe, T. J., Brockington, S., Case, A., Cassibry, J. T., Kagan, G., Messer, S. J., StAnic, M., TANG, X., Welch, D. R., et al. 2012a Spherically imploding plasma liners as a standoff driver for magnetoinertial fusion. IEEE Trans. Plasma Sci. 40, 1287.

Hsu, S. C. \& BELlan, P. M. 2005 On the jets, kinks, and spheromaks formed by a planar magnetized coaxial gun. Phys. Plasmas 12, 032103.

HSU, S. C. \& LANGENDORF, S. J. 2019 Magnetized plasma target for plasma-jet-driven magneto-inertial fusion. J. Fusion Energy 38, 182.

Hsu, S. C., Langendorf, S. J., Yates, K. C., Dunn, J. P., Brockington, S., Case, A., Cruz, E., Witherspoon, F. D., Gilmore, M. A., Cassibry, J. T., et al. 2018 Experiment to form and characterize a section of a spherically imploding plasma liner. IEEE Trans. Plasma Sci. 46, 1951.

Hsu, S. C., Merritt, E. C., Moser, A. L., Awe, T. J., Brockington, S. J. E., Davis, J. S., Adams, C. S., Case, A., Cassibry, J. T., Dunn, J. P., et al. $2012 b$ Experimental characterization of Railgun-driven supersonic plasma jets motivated by high energy density physics applications. Phys. Plasmas 19, 123514.

Hutchinson, I. H. 2002 Principles of Plasma Diagnostics, 2nd edn. Cambridge University Press.

Kadomtsev, B. B. \& Pogutse, O. P. 1978 Plasma electron thermal conductivity across a braided magnetic field. In 7th IAEA Conference on Plasma Physics and Controlled Nuclear Fusion Research, pp. 23-30. I. V. Kurchatov Inst. of Atomic Energy. Moscow.

KirkPATRICK, R. C., Lindemuth, I. R. \& WARD, M. S. 1995 Magnetized target fusion: an overview. Fusion Technol. 27, 201.

Kulsrud, R. M. \& Zweibel, E. G. 2008 On the origin of cosmic magnetic fields. Rep. Prog. Phys. 71, 046901.

Kunz, M., Squire, J., Balbus, S., Bale, S., Chen, C., Churazov, E., Cowley, S., Forest, C., GAMmie, C., QUATAERT, E., et al. 2020 The material properties of weakly collisional, high- $\beta$ plasmas. arXiv:1903.04080. White Paper for the Plasma 2020 Decadal Survey.

Langendorf, S. J., Yates, K. C., Hsu, S. C., Thoma, C. \& Gilmore, M. 2019 Experimental study of ion heating in obliquely merging hypersonic plasma jets. Phys. Plasmas 26, 082110.

LiNDEMUth, I. R. \& SiEMON, R. E. 2009 The fundamental parameter space of controlled thermonuclear fusion. Am. J. Phys. 77, 407.

Macfarlane, J., Golovkin, I. E., Woodruff, P. R., Welch, D. R., Oliver, B. V., Melhorn, T. A. \& CAMPBell, R. B. 2004 Simulation of the ionization dynamics of aluminum irradiated by intense short-pulse lasers. In Proc. Inertial Fusion and Sciences Applications 2003 (ed. B. A. Hammel, D. D. Meyerhofer \& J. Meyer-ter Vehn), p. 457. American Nuclear Society.

Matsumoto, T., Roche, T., Allfrey, I., Sekiguchi, J., Asai, T., Gota, H., Cordero, M., Garate, E., Kinley, J., Valentine, T., et al. 2016a Characterization of compact-toroid injection during formation, translation, and field penetration. Rev. Sci. Instrum. 87, $11 \mathrm{D} 406$.

Matsumoto, T., Sekiguchi, J., Asai, T., Gota, H., Garate, E., Allfrey, I., Valentine, T., Morehouse, M., Roche, T., Kinley, J., et al. $2016 b$ Development of a magnetized coaxial plasma gun for compact toroid injection into the C-2 field-reversed configuration device. Rev. Sci. Instrum. 87, 053512.

McKee, C. F. \& Ostriker, E. C. 2007 Theory of star formation. Annu. Rev. Astron. Astrophys. 45 (1), $565-687$.

Merritt, E. C., Moser, A. L., Hsu, S. C., Adams, C. S., Dunn, J. P., Holgado, A. M. \& Gilmore, M. A. 2014 Experimental evidence for collisional shock formation via two obliquely merging supersonic plasma jets. Phys. Plasmas 21, 055703.

Messer, S., Case, A., Wu, L., Brockington, S. \& Witherspoon, F. D. 2013 Nonlinear compressions in merging plasma jets. Phys. Plasmas 20 (3), 032306.

Moser, A. L. \& HSU, S. C. 2015 Experimental characterization of a transition from collisionless to collisional interaction between head-on-merging supersonic plasma jets. Phys. Plasmas 22, 055707.

Ono, Y., Inomoto, M., Ueda, Y., Matsuyama, T. \& OKAZAKi, T. 1999 New relaxation of merging spheromaks to a field reversed configuration. Nucl. Fusion 39, 2001. 
Peterson, J. R. \& Fabian, A. C. 2006 X-ray spectroscopy of cooling clusters. Phys. Rep. 427, 1.

Rechester, A. B. \& Rosenbluth, M. N. 1978 Electron heat transport in a tokamak with destroyed magnetic surfaces. Phys. Rev. Lett. 40 (1), 38-41.

RYUTOV, D. D. 2009 Adiabatic compression of a dense plasma "mixed" with random magnetic fields. Fusion Sci. Technol. 56, 1489.

Ryutov, D. D., Kane, J. O., Mizuta, A., Pound, M. W. \& Remington, B. A. 2004 Two models of magnetic support for photoevaporated molecular clouds. In High Energy Density Laboratory Astrophysics (ed. G. A. Kyrala), pp. 183-190. Springer.

Ryutov, D. D. \& Remington, B. A. 2002 Scaling astrophysical phenomena to high-energy-density laboratory experiments. Plasma Phys. Control. Fusion 44, B407.

Scalo, J. \& Elmegreen, B. G. 2004 Interstellar turbulence II: implications and effects. Annu. Rev. Astron. Astrophys. 42 (1), 275-316.

SCHeKochinin, A. A. \& Cowley, S. C. 2007 Turbulence and magnetic fields in astrophysical plasmas. In Magnetohydrodynamics. Fluid Mechanics And Its Applications (ed. Sergei Molokov, René Moreau \& Keith Moffatt), vol. 80, pp. 85-115. Springer.

Schekochihin, A. A., Cowley, S. C., Dorland, W., Hammett, G. W., Howes, G. G., QuATAERT, E. \& TATSUnO, T. 2009 Astrophysical gyrokinetics: kinetic and fluid turbulent cascades in magnetized weakly collisional plasmas. Astrophys. J. Suppl. Ser. 182, 310.

Schekochinin, A. A., Cowley, S. C., Kulsrud, R. M., Hammett, G. W. \& Sharma, P. 2005 Plasma instabilities and magnetic field growth in clusters of galaxies. Astrophys. J. 629, 139.

Schekochinin, A. A., Cowley, S. C., Kulsrud, R. M., Rosin, M. S. \& Heinemann, T. 2008 Nonlinear growth of firehose and mirror fluctuations in astrophysical plasmas. Phys. Rev. Lett. 100, 081301.

Slough, J., Votroubek, G. \& Pinl, C. 2011 Creation of a high-temperature plasma through merging and compression of supersonic field reversed configuration plasmoids. Nucl. Fusion 51, 053008.

Slutz, S. A., Herrmann, M. C., Vesey, R. A., Sefkow, A. B., Sinars, D. B., Rovang, D. C., Peterson, K. J. \& Cuneo, M. E. 2010 Pulsed-power-driven cylindrical liner implosions of laser preheated fuel magnetized with an axial field. Phys. Plasmas 17, 056303.

SmiRnov, B. M. 2000 Tables for cross sections of the resonant charge exchange process. Phys. Scr. 61 (5), 595-602.

Steinhauer, L. C. 2011 Review of field-reversed configurations. Phys. Plasmas 18, 0070501.

Thio, Y. C. F., Hsu, S. C., Witherspoon, F. D., Cruz, E., Case, A., Langendorf, S., Yates, K., Dunn, J., CAssibry, J., SAmulyak, R., et al. 2019 Plasma-jet-driven magneto-inertial fusion. Fusion Sci. Technol. 75, 581.

Thio, Y. C. F., Panarella, E., Kirkpatrick, R. C., Knapp, C. E., Wysocki, F., Parks, P. \& SCHMIDT, G. 1999 Magnetized target fusion in a spheroidal geometry with standoff drivers. In Current Trends in International Fusion Research-Proceedings of the Second International Symposium (ed. E. Panarella), p. 113. NRC Canada.

Yamada, M., Ono, Y., Hayakawa, A., Katsurai, M. \& Perkins, F. W. 1990 Magnetic reconnection of plasma toroids with cohelicity and counterhelicity. Phys. Rev. Lett. 65, 721.

Yates, K. C., Langendorf, S. J., Hsu, S. C., Dunn, J. P., Brockington, S., Case, A., Cruz, E., Witherspoon, F. D., Thio, Y. C. F., CAssibry, J. T., et al. 2020 Experimental characterization of a section of a spherically imploding plasma liner formed by merging hypersonic plasma jets. Phys. Plasmas 27, 062706.

YEE, J. \& BELLAN, P. M. 2000 Taylor relaxation and $\lambda$ decay of unbounded, freely expanding spheromaks. Phys. Plasmas 7, 3625. 